\title{
Giant Left Main Coronary Artery Aneurysm Presenting as Multiple Implantable Cardioverter Defibrillator Shocks
}

\author{
Yury Malyshev $^{1}$, Asma Syed ${ }^{2}$, Ricardo Castillo ${ }^{2}$, Rumman A. Syed ${ }^{3}$, Sonu Sahni ${ }^{3,4,5}$ \\ 1. Cardiology, Maimonides Medical Center, Brooklyn, USA 2. Cardiology, Brookdale University Hospital Medical \\ Center, Brooklyn, USA 3. Internal Medicine, Brookdale University Hospital Medical Center, Brooklyn, USA 4. Research \\ Medicine, New York Institute of Technology College of Osteopathic Medicine, New York, USA 5. Primary Care, Touro \\ College of Osteopathic Medicine, New York, USA
}

Corresponding author: Yury Malyshev, yurimalish@hotmail.com

\begin{abstract}
Giant aneurysms of the left main coronary artery are one of the rarest findings in cardiology, encountered in less than $0.02 \%$ of patients. The presentation is usually the same as coronary artery disease since most coronary aneurysms in the western world are associated with atherosclerosis. Here we report the first case of giant aneurysm of the left main coronary artery presenting as ventricular tachycardia with multiple shocks of the defibrillator in a 57-year-old man with heart failure. We also review the etiology, pathology, and management of coronary aneurysms.
\end{abstract}

Received 03/17/2020

Review began 03/28/2020 Review ended 04/05/2020 Published 04/12/2020

() Copyright 2020

Malyshev et al. This is an open access article distributed under the terms of the Creative Commons Attribution License CC-BY 4.0., which permits unrestricted use, distribution, and reproduction in any medium, provided the original author and source are credited.
Categories: Cardiology, Anatomy

Keywords: coronary artery aneurysm, defibrillator shock, ventricular tachycardia, left main coronary artery aneurysm, left main aneurysm

\section{Introduction}

Coronary artery aneurysms (CAAs) are very rare clinical entities; among them giant left main coronary artery aneurysms (LMCAAs) are exceedingly rare, encountered in less than $0.02 \%$ of patients [1]. Etiology of CAA varies depending on age, comorbidities and even geographical area. The etiology usually determines presentation and management. Herein we report a unique case of giant LMCAA in a 57-year-old man with heart failure with reduced ejection fraction (HFrEF), who presented to our emergency department with chest pain after his implantable cardioverter defibrillator (ICD) fired 12 times. Urgent diagnostic catheterization showed giant LMCAA without signs of coronary artery disease (CAD). The patient was started on dual antiplatelet therapy. He remained asymptomatic for more than a year. We also review current literature on various diagnostic modalities and different management approaches of CAAs.

\section{Case Presentation}

A 57-year-old man with hypertension, diabetes, obesity, and HFrEF presented with chest pain, palpitations, and syncope the day before. His ICD fired 12 times. His vitals were unremarkable. Physical examination was significant for irregular pulse. EKG showed normal sinus rhythm with frequent premature ventricular complexes and left anterior fascicular block (Figure 1).

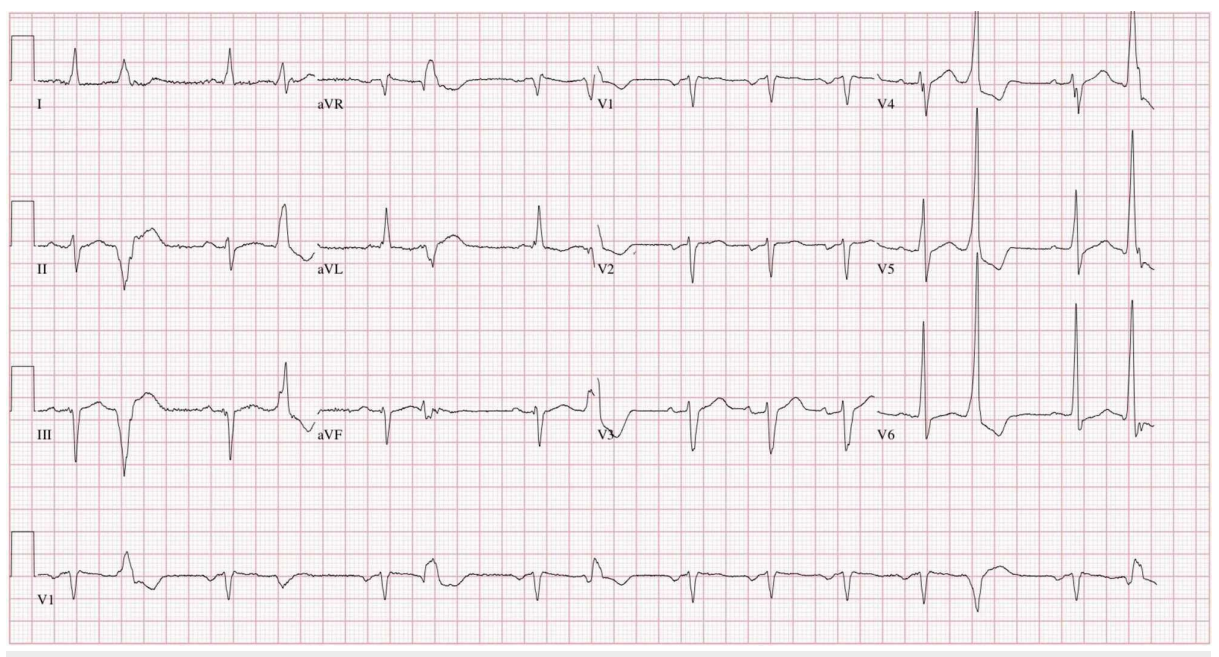

FIGURE 1: EKG showing sinus rhythm with premature ventricular 


\section{Cureus}

contractions and left anterior fascicular block

Chest x-ray was clear. Blood work showed elevated cardiac enzymes and electrolyte abnormality, and drug screen was positive for cannabinoid (Table 1).

\begin{tabular}{|l|l}
\hline Test & Result \\
\hline Troponin $(\mathrm{ng} / \mathrm{mL})$ & 2.14 \\
CPK (U/L) & 537 \\
pBNP $(\mathrm{pg} / \mathrm{mL})$ & 1860 \\
Potassium $(\mathrm{mEq} / \mathrm{L})$ & 2.8 \\
\hline ANA & Negative \\
Anti-dsDNA antibody (IU/mL) & 1 \\
pANCA (AU/mL) & $<1.0$ \\
C3 complement $(\mathrm{mg} / \mathrm{dL})$ & 202 \\
C4 complement $(\mathrm{mg} / \mathrm{dL})$ & 40 \\
Drug screen & Cannabinoids
\end{tabular}

\section{TABLE 1: Significant lab results}

CPK, creatine phosphokinase; pBNP, pro-brain natriuretic peptide; ANA, antinuclear antibody; anti-dsDNA, anti-double-stranded DNA antibody; pANCA, perinuclear antineutrophil cytoplasmic antibodies

ICD interrogation showed that two shocks were administered for ventricular tachycardia and 10 shocks were inappropriate due to electromagnetic interference on the lead. Echocardiogram revealed ejection fraction of $10 \%-15 \%$ with diffuse hypokinesis (Video 1).

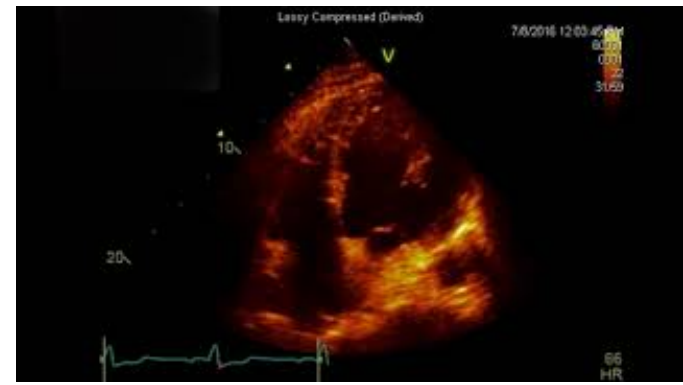

VIDEO 1: Echocardiogram showing severely reduced left ventricular systolic function, ejection fraction of $10 \%-15 \%$, and diffuse hypokinesis

View video here: https://www.youtube.com/watch?v=XlyaB9tamJ8

Urgent cardiac catheterization showed no evidence of occlusive CAD. There was however a large saccular LMCAA involving the ostium of the left anterior descending (LAD), left circumflex (LCX), and ramus intermedius arteries. The size of the aneurysm was measured to be $37.4 \mathrm{~mm}$ x $20 \mathrm{~mm}$ (Figure 2, Video 2). Autoimmune workup was negative (Table 1). 


\section{Cureus}

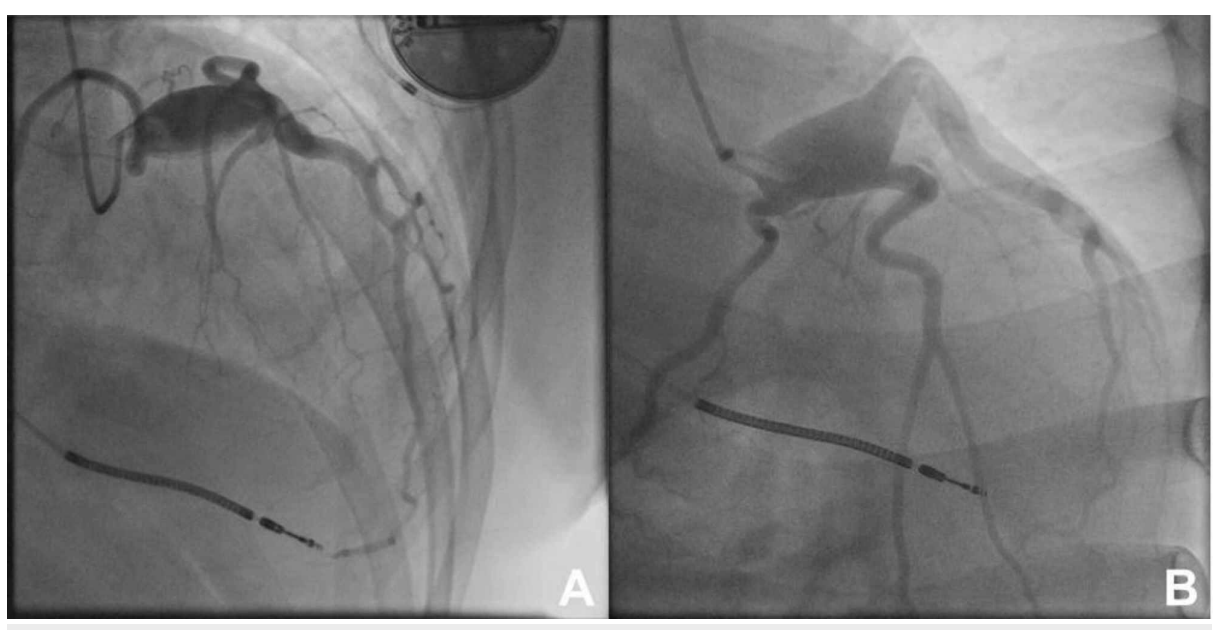

FIGURE 2: Giant aneurysm of the left main coronary aneurysm

A: right anterior oblique cranial view; B: right anterior oblique caudal view

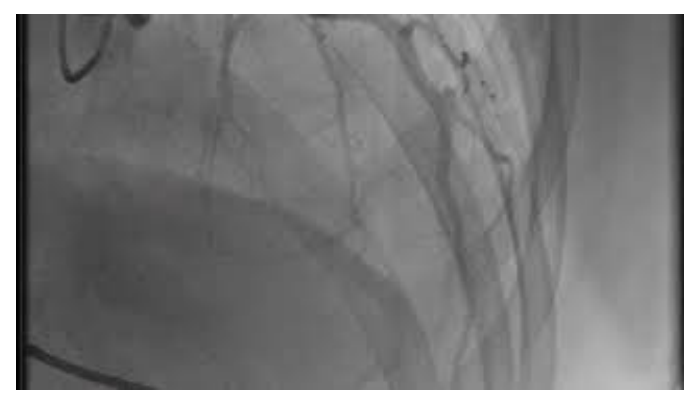

VIDEO 2: Angiogram showing giant aneurysm of the left main coronary artery

View video here: https://www.youtube.com/watch?v=A8vu-i-HNAg

The patient was started on dual antiplatelet therapy with aspirin and clopidogrel. CT surgery evaluated the patient, but did not recommend intervention. The patient had successful implantation of a cardioverter defibrillator during the same admission. He was seen in ED one year later with suspicion for pulmonary embolism. CT chest angiogram showed LMCAA to be $1.5 \mathrm{~cm}$ in diameter (Figure 3).

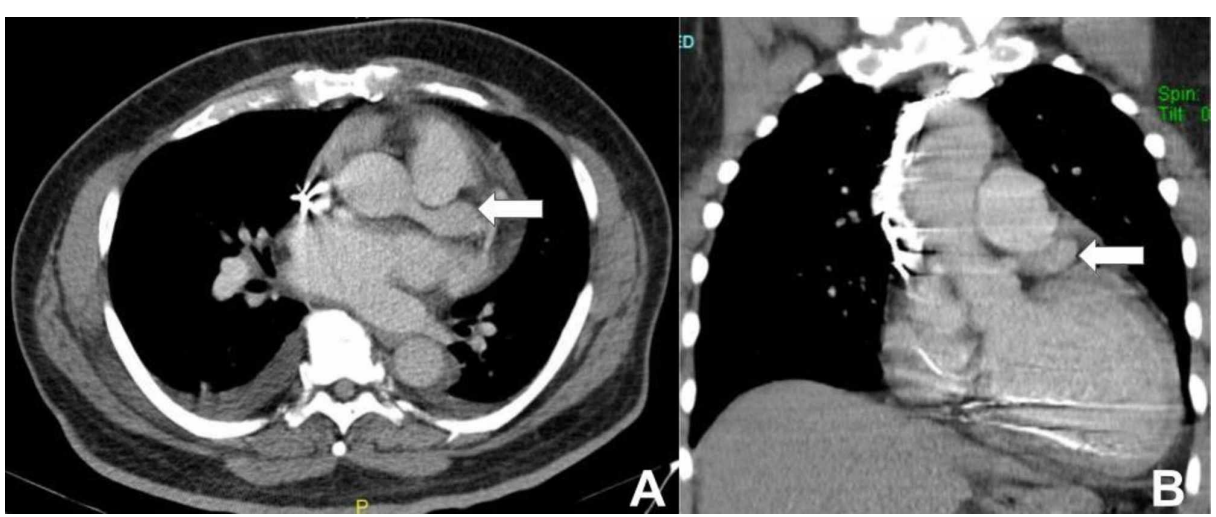

FIGURE 3: CT chest showing left main coronary artery aneurysm (white arrow)

A: transverse plane; B: coronal plane 


\section{Cureus}

Two months after the ED visit, he was seen in the clinic symptom free and ICD was functioning well.

\section{Discussion}

CAA is a segment of the artery with width greater than length and diameter greater than diameter of a normal adjacent segment or 1.5 times larger than the largest coronary vessel (Figure 4) [2,3].

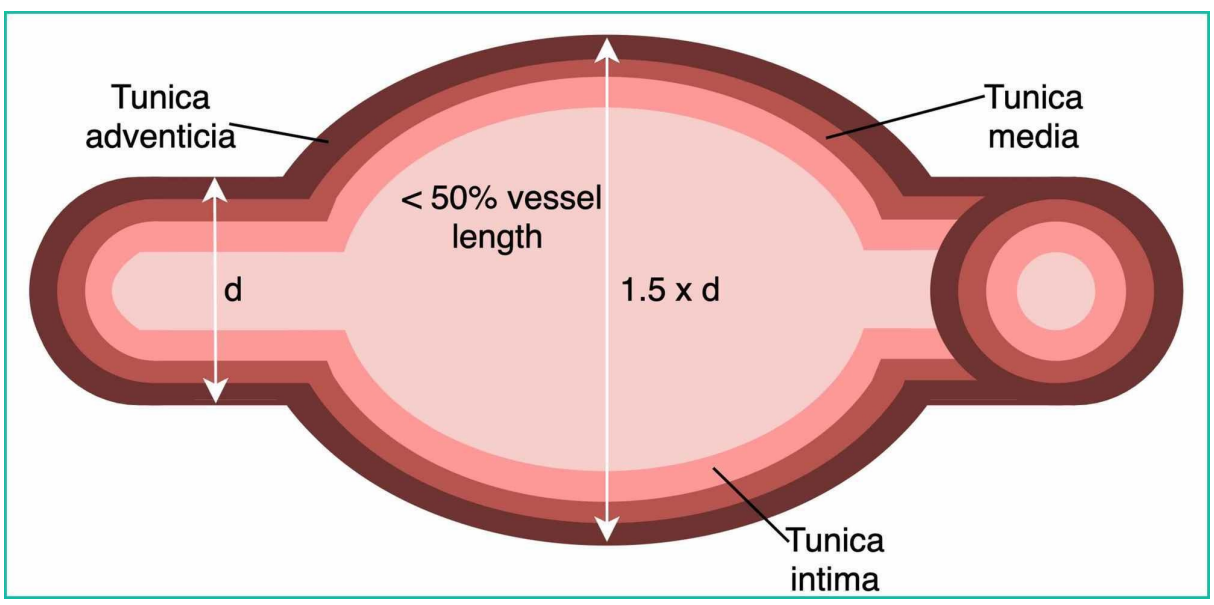

FIGURE 4: Schematic representation of a true coronary aneurysm

d, diameter

CAAs are classified as follows. Wall composition: true aneurysms have all three vessel wall layers; pseudoaneurysms lose one or two. Shape: saccular CAAs transverse diameter is greater than the longitudinal diameter. They are often seen distal to stenosis and are more prone to thrombosis or rupture. Fusiform aneurysms involve the whole vessel circumference, have greater longitudinal measurement, and have no relationship to stenosis. Size: small (diameter $<5 \mathrm{~mm}$ ), medium $(5-8 \mathrm{~mm})$, and giant $(>8 \mathrm{~mm})$ [3].

Incidence varies from $0.3 \%$ to $5.3 \%$ (mean of $1.65 \%$ ). Men have more CAAs than women: $2.2 \%$ vs. $0.5 \%$. Most frequent locations are right coronary (40\%-70\%), LCX (23.4\%), and LAD (32.3\%) arteries. Left main coronary artery is affected significantly less (0.1\%-3.5\%) (Figure 5) [4,5]. Prevalence of giant CAA in a general population is only $0.02 \%[1]$.

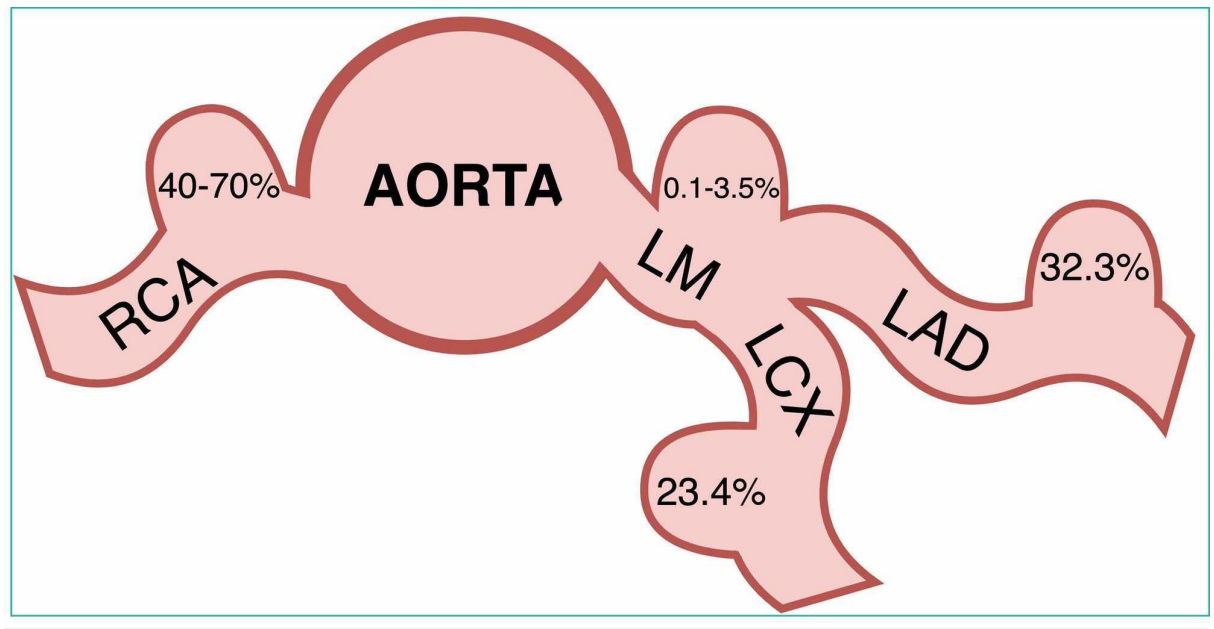

FIGURE 5: Distribution and fréquence of coronary artery aneurysms

LM, left main coronary artery; LAD, left anterior descending artery; LCX, left circumflex artery; RCA, right coronary artery

The etiology of CAA varies depending on age and geographical area. Atherosclerosis is responsible for half of CAAs in the West, followed by congenital (17\%) and infectious (10\%) aneurysms. Kawasaki disease is the dominant cause of CAA in Japan [3]. Inflammatory disorders and connective tissue diseases are usually 
associated with ectasias and are more frequent in younger patients [3]. Iatrogenic causes include trauma from balloon inflation pressure, intervention in acute myocardial infarction, use of nonsteroidal antiinflammatory drugs, steroids, and colchicine, which can cause improper healing. Cocaine causes severe hypertension and vasoconstriction, thus damaging the endothelium and promoting CAA formation [6].

CAAs in CAD are thought to be caused by turbulent blood flow damaging the wall [3]. However, there must be other factors because most patients with CAD do not develop CAA.

Usually patients are asymptomatic and most CAAs are found incidentally. Presentation depends on the etiology and/or complications. Complications of CAA include embolization, rupture, fistula formation, tamponade, hemopericardium, dissection, vasospasm, and vessel compression $[2,3]$.

Coronary angiography remains the best method to identify CAA [2]. It provides information about location, size, and shape of CAA, but it only sees the vessel lumen. Thus, the true size of CAA could be underestimated, or CAA can be missed if thrombus occludes it [4]. Intravascular ultrasound corrects these limitations, providing transmural images and information about wall structure and luminal composition $[4,7]$. CT coronary angiography provides fast information about CAA's location, shape, size, and wall composition, but no treatment option. CT angiography is useful in following patients with known CAAs [4].

Management of CAA depends on presentation, etiology, size, location, associated infection, and extent of atherosclerosis [4]. In adults with $\mathrm{CAD}$, medical reduction of cardiovascular risk factors should be started. Long-term antiplatelets and potentially anticoagulation should be started since thrombosis and/or embolism are of concern [8]. Percutaneous intervention with stent placement can be done in aneurysm with diameter up to $10 \mathrm{~mm}$ [9]. Surgery is indicated in patients, who are not candidates for percutaneous intervention, obstructive $\mathrm{CAD}$, and large saccular aneurysms at risk for rupture $[4,10]$.

\section{Conclusions}

The first case of CAA was published in 1812. It was found post-mortem after sudden death. Since then our understanding of the pathology, etiology, and progression of CAAs has improved. Today we can find these potentially deadly aneurysms during routine angiogram, not post-mortem. However, their management is still challenging and has to be tailored specifically to each patient. More research is needed to identify patients, who are at risk to diagnose CAA earlier, manage it better, and prevent complications.

\section{Additional Information \\ Disclosures}

Human subjects: Consent was obtained by all participants in this study. Conflicts of interest: In compliance with the ICMJE uniform disclosure form, all authors declare the following: Payment/services info: All authors have declared that no financial support was received from any organization for the submitted work. Financial relationships: All authors have declared that they have no financial relationships at present or within the previous three years with any organizations that might have an interest in the submitted work. Other relationships: All authors have declared that there are no other relationships or activities that could appear to have influenced the submitted work.

\section{References}

1. Li D, Wu Q, Sun L, et al.: Surgical treatment of giant coronary artery aneurysm. J Thorac Cardiovasc Surg. 2005, 130:817-821. 10.1016/j.jtcvs.2005.04.004

2. Pahlavan PS, Niroomand F: Coronary artery aneurysm: a review. Clin Cardiol. 2006, 29:439-443. https://doi.org/10.1002/clc.4960291005

3. Diaz-Zamudio M, Bacilio-Perez U, Herrera-Zarza MC, et al.: Coronary artery aneurysms and ectasia: role of coronary CT angiography. Radiographics. 2009, 29:1939-1954. 10.1148/rg.297095048

4. Abou Sherif S, Ozden Tok O, Taskoylu O, Goktekin O, Kilic ID: Coronary artery aneurysms: a review of the epidemiology, pathophysiology, diagnosis, and treatment. Front Cardiovasc Med. 2017, 4:24. 10.3389/fcvm.2017.00024

5. Swaye PS, Fisher LD, Litwin P, et al.: Aneurysmal coronary artery disease. Circulation. 1983, 67:134-138. 10.1161/01.cir.67.1.134

6. Satran A, Bart BA, Henry CR, et al.: Increased prevalence of coronary artery aneurysms among cocaine users . Circulation. 2005, 111:2424-2429. 10.1161/01.CIR.0000165121.50527.DE

7. Porto I, MacDonald S, Banning AP: Intravascular ultrasound as a significant tool for diagnosis and management of coronary aneurysms. Cardiovasc Intervent Radiol. 2004, 27:666-668. 10.1007/s00270-0040038-0

8. Nichols L, Lagana S, Parwani A: Coronary artery aneurysm: a review and hypothesis regarding etiology . Arch Pathol Lab Med. 2008, 132:823-828. https://doi.org/10.1043/1543-2165(2008)132[823:CAAARA]2.0.CO;2

9. Szalat A, Durst R, Cohen A, Lotan C: Use of polytetrafluoroethylene-covered stent for treatment of coronary artery aneurysm. Catheter Cardiovasc Interv. 2005, 66:203-208. 10.1002/ccd.20448

10. LaMotte LC, Mathur VS: Atherosclerotic coronary artery aneurysms: eight-year angiographic follow-up. Tex Heart Inst J. 2000, 27:72-73. 